\title{
Expression of cotton PLATZ1 in transgenic Arabidopsis reduces sensitivity to osmotic and salt stress for germination and seedling establishment associated with modification of the abscisic acid, gibberellin, and ethylene signalling pathways
}

Shicai Zhang ${ }^{\dagger}$, Rui Yang ${ }^{\dagger}$, Yanqing Huo, Shasha Liu, Guodong Yang, Jinguang Huang, Chengchao Zheng and Changai $\mathrm{Wu}^{*}$

\begin{abstract}
Background: Zinc-finger transcription factors play central roles in plant growth, development and abiotic stress responses. PLATZ encodes a class of plant-specific zinc-finger transcription factor. However, biological functions or physiological mechanism controlled by PLATZ are currently limited.

Results: GhPLATZ1 transcripts were considerably up-regulated by $\mathrm{NaCl}$, mannitol, abscisic acid (ABA) and gibberellin (GA) treatments. Transgenic Arabidopsis by ectopic expression of GhPLATZ1 exhibited faster seed germination and higher seedling establishment under salt and mannitol stresses than those of wild type (WT), indicating enhanced osmotic insensitivity in GhPLATZ1 transgenic Arabidopsis. The ABA content in dry seeds of GhPLATZ1 transgenic Arabidopsis was lower than that of WT whereas the ABA content was not changed in germinating seeds under salt stress. Seed germination was faster than but the seedling establishment of transgenic Arabidopsis was similar to WT. Besides, GhPLATZ1 transgenic and WT Arabidopsis exhibited insensitivity to paclobutrazol (PAC), a GA biosynthesis inhibitor, whereas exogenous GA could eliminate the growth difference between GhPLATZ1 transgenic and WT Arabidopsis under salt stress. Moreover, exogenous 1-aminocyclopropane-1-carboxylic acid (ACC), an ethylene precursor, exerted similar effects to GA. Furthermore, ABI4 and ETO1 transcripts were significantly down-regulated, whereas ACS8 was up-regulated in GhPLATZ1 transgenic Arabidopsis under salt stress.
\end{abstract}

Conclusions: In conclusion, GhPLATZ1 had broad influence in responses to salt and mannitol stresses in transgenic Arabidopsis during seed germination and seedling establishment. The effect of GhPLATZ1 expression in transgenic Arabidopsis might be mediated by the ABA, GA, and ethylene pathways. Thus, this study provided new insights into the regulatory network in response to abiotic stresses in plants.

Keywords: Gossypium hirtusum L, PLATZ, Abiotic stresses, Ethylene, ABA, GAs

\footnotetext{
* Correspondence: cawu@sdau.edu.cn

'Shicai Zhang and Rui Yang contributed equally to this work.

State Key Laboratory of Crop Biology, College of Life Sciences, Shandong

Agricultural University, Tai'an, Shandong, People's Republic of China
}

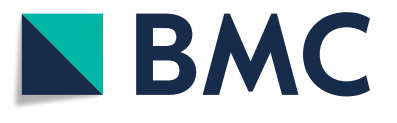

(c) The Author(s). 2018 Open Access This article is distributed under the terms of the Creative Commons Attribution 4.0 International License (http://creativecommons.org/licenses/by/4.0/), which permits unrestricted use, distribution, and reproduction in any medium, provided you give appropriate credit to the original author(s) and the source, provide a link to the Creative Commons license, and indicate if changes were made. The Creative Commons Public Domain Dedication waiver (http://creativecommons.org/publicdomain/zero/1.0/) applies to the data made available in this article, unless otherwise stated. 


\section{Background}

Impaired germination and postgermination growth are common among seeds exposed to salt and drought stresses. Plants can regulate and coordinate both growth and/or stress sensitivity to promote survival or escape from environmental stress by modifying the production, distribution, or signal transduction of hormones. Thus, identification of components involved in hormonal regulation during germination under abiotic stresses is essential.

A large number of genes encoding DNA-binding proteins, which play important roles in hormone mediated responses to abiotic stresses were identified in plants. For example, the major downstream components of abscisic acid (ABA) signaling, namely, ABA insensitive 3 (ABI3) [1], ABI4 [2] and ABI5 [3, 4], are positive regulators of $\mathrm{ABA}$, and they are crucial in seed maturation, seed germination and seedling growth [5]. Fuctional loss of these genes causes fast germination under ABA and abiotic stresses. The GA signaling pathways depend on DELLA proteins including GA insensitive ones (GAI), repressor of ga1-3 (RGA), repressor of ga13-like (RGL) 1, RGL2 and RGL3 [6, 7]. GA destabilizes the DELLA proteins, which act as growth repressors, by targeting ubiquitination and degradation [8]. EIN2 plays a key role in ethylene signaling and losing its function results in a hypersensitivity to salt and osmotic stress during germination and early seedling development in Arabidopsis [9]. ERF genes might also play a pivotal role in ethylene responsiveness and germination regulation in tomato (Solanum lycopersicon); SlERF2 overexpression in transgenic lines causes premature seed germination [10].

Zinc-finger transcription factors are a relatively large family of plant transcription factors (approximately 15\% of the total) and play central roles in plant growth and development $[11,12]$. PLATZ1 was first isolated from pea [13]. PLATZ1 and its paralogs from Arabidopsis and other species have two consensus signatures, namely, $\mathrm{C}-\mathrm{x}_{2}-\mathrm{H}-\mathrm{x}_{11}-\mathrm{C}-\mathrm{x}_{2}-\mathrm{C}-\mathrm{x}_{(4-5)}-\mathrm{C}-\mathrm{x}_{2}-\mathrm{C}-\mathrm{x}_{(3-7)}-\mathrm{H}-\mathrm{x}_{2}-\mathrm{H} \quad\left(\mathrm{CHC}_{4} \mathrm{H}_{2}\right)$ and $\mathrm{C}-\mathrm{x}_{2}-\mathrm{C}-\mathrm{x}_{(10-11)}-\mathrm{C}-\mathrm{x}_{3}-\mathrm{C}$. However, the two consensus signatures are different from those of previously characterized zinc-binding motifs, such as RING $\left(\mathrm{C}_{3} \mathrm{HC}_{4}\right)$, LIM $\left(\mathrm{C}_{2} \mathrm{HC}_{5}\right)$ [14], GATA finger $\left.\left(\mathrm{C}-\mathrm{x}_{2}-\mathrm{C}-\mathrm{x}_{(17-18)}\right)^{-} \mathrm{C}-\mathrm{x}_{2}-\mathrm{C}\left(\mathrm{C}_{2} \mathrm{C}_{2}\right)\right)$ $[15,16]$, COSTANS/CONSTANS-like (CO/COLs) [17], and the DNA-binding one finger (Dof) [18]. Hence PLATZs encode a novel class of plant-specific zinc-finger transcription factors. Recently, Arabidopsis PLATZ1 and 2 were reported to positively regulate acquisition of dessication tolerance in seeds and vegetative tissues [19]. Maize PLATZ protein FL3 is involved in tRNA and 5S rRNA transcription through interaction with RNA polymerase III [20]. Multiple members of PLATZ genes exist in plants. For example, 12 PLATZ genes exist in the Arabidopsis genome (https://www.arabidopsis.org/ servlets/Search?type=general\&search_action=detail $\&$ method $=1 \&$ show_obsolete $=$ F\&name $=$ PLATZ\&sub_type $=$ gene\&SEARCH_EXACT=4\&SEARCH_CONTAINS=1/). Therefore, the roles of PLATZs remain largely unclear.

Cotton (Gossypium hirsutum) is one of the most important fibre and oil crops. Cotton seed germination and seedling establishment are severely impaired in abiotic stress conditions. The biological significance of cotton PLATZs has not yet been described. In this study, the first PLATZ form cotton, named as GhPLATZ1, was isolated and characterized. Our results indicated that the expression of GhPLATZ1 was induced by abiotic and hormone stimuli. Ectopic expression of GhPLATZ1 in Arabidopsis resulted in enhanced insensitivity to osmotic stresses, ABA and PAC. We deduced that GhPLATZ1 might play important roles in regulating hormonemediated osmotic stress during cotton seed germination and seedling establishment.

\section{Results}

Characterization of GhPLATZ1Transcription factor

We previously showed that a gene (GenBank ID: JQ837703) from cotton was significantly induced by salt stress [21]. The deduced amino acid sequence was homologous to PLATZ1 from peas and PLATZs from Arabidopsis. Given that the gene was the first isolated PLATZ from cotton, we named it as GhPLATZ1. Genome-wide searching in G.hirtusium and Arabidopsis thaliana revealed that 9 PLATZ genes exist in cotton (https://phytozome.jgi. doe.gov) and 12 are in Arabidopsis [the Arabidopsis Information Resource (TAIR), https://www.arabidopsis. org $/$ servlets $/$ Search?type=general\&search_action $=$ detail $\&$ method=1\&show_obsolete $=$ F $\&$ name $=$ PLATZ\&sub_type $=$ gene\&SEARCH_EXACT $\left.=4 \& S E A R C H \_C O N T A I N S=1\right]$. GhPLATZs showed high identity with each other $(\geq 79.56 \%)$ (Additional file 1: Figure S1A), and all of them shared numerous similarities to a subfamily of AtPLATZs, including AtPLATZ3, AtPLATZ11, and AtPLATZ12 (Additional file 1: Figure S1B). However, the roles of GhPLATZs remain unclear.

To explore the possible roles of GhPLATZ1 under salt stress and other abiotic stresses, the expression of GhPLATZ1 was determined in 20-day-old seedlings by qRT-PCR using GhUBI (EU604080) as the reference gene. GhPLATZ1 transcripts were induced by $3.8,6.2,6.8$, or 3.6-fold by $\mathrm{NaCl}$, mannitol, $\mathrm{GA}$, or ABA treatment, respectively (Fig. 1a). During seed germination, GhPLATZ1 transcripts were up-regulated by 4.1 and 7.2-fold or 7.5 and 4.5-fold by $\mathrm{NaCl}$ or mannitol treatment at 6 and $12 \mathrm{~h}$, respectively, or two fold by GA and ABA treatments at $6 \mathrm{~h}$ (Fig. 1b). In addition, high levels of GhPLATZ1 transcripts were detected in roots, stems and cotyledons, whereas low transcripts were found in leaves and seeds 

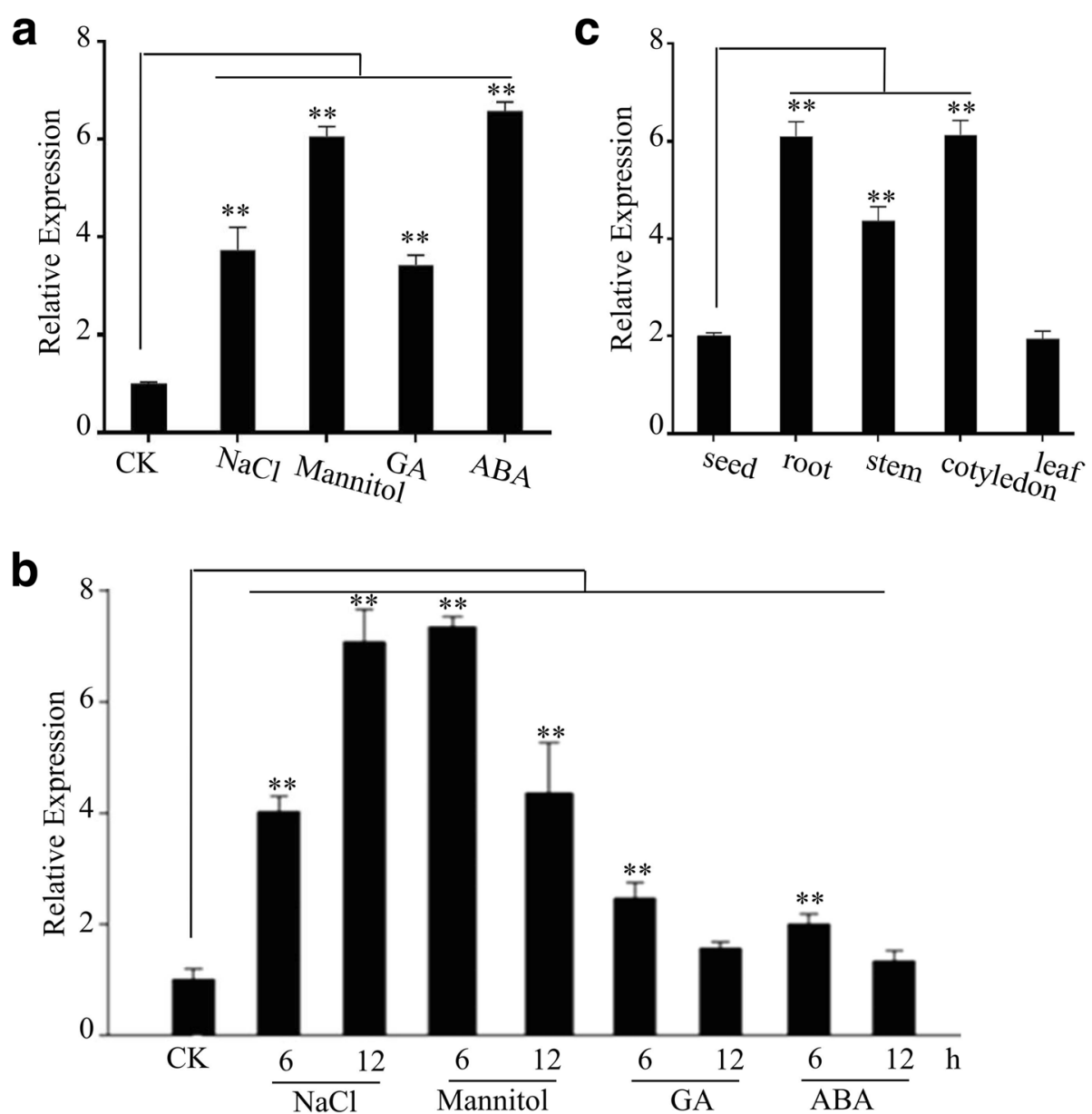

Fig. 1 The expression patterns of GhPLATZ1 analyzed by qRT-PCR. a GhPLATZ1 expression in 20-day-old seedlings treated with or without $200 \mathrm{mM}$ $\mathrm{NaCl}, 300 \mathrm{mM}$ mannitol, $50 \mu \mathrm{M}$ GA or $50 \mu \mathrm{M}$ ABA for $6 \mathrm{~h}$. b GhPLATZ1 expression in 2.5-day germinated seeds in Hoagland solutions with or without $200 \mathrm{mM} \mathrm{NaCl}, 300 \mathrm{mM}$ mannitol, $50 \mu \mathrm{M} \mathrm{GA}$ or $50 \mu \mathrm{M}$ ABA for 6 and $12 \mathrm{~h}$. The transcript levels were normalized to that of GhUBI (EU604080). Values are means \pm SD of three replicates. c GhPLATZ1 expression in seeds, roots, stems, cotyledens or leaves of 20-day-old cotton seedlings. At least three independent experiments were conducted

(Fig. 1c). These data suggested that GhPLATZ1may be involved in plant responses to environmental stimuli.

\section{GhPLATZ1 transgenic Arabidopsis enhances insensitivity to osmotic stresses}

To further analyze the function of GhPLATZ1, we introduced 35S::GhPLATZ1 into Arabidopsis, and obtained three independent transgenic lines with high levels of GhPLATZ1 transcripts (Fig. 2a) for further analysis.

When seeds of WT and three transgenic lines were sown on $1 / 2$ MS medium, the three transgenic lines showed similar seed germination and seedling establishment to WT did (Fig. 2b and c; Additional file 1: Figures. S2 and S3). However, when subjected to $1 / 2 \mathrm{MS}$ medium with $175 \mathrm{mM} \mathrm{NaCl}$, the three transgenic lines exhibited more than $20 \%$ higher germination rates than WT during 2.5-4 days, and reached the maximum germination rate 1.5 days earlier than WT (Fig. 2b). Cotyledon greening was also more than $20 \%$ higher in the transgenic lines compared to WT (Fig. 2c and d). Similar results were obtained when seeds of WT and three transgenic lines were subjected to $1 / 2 \mathrm{MS}$ medium with 150 and $200 \mathrm{mM} \mathrm{NaCl}$ (Additional file 1: Figures. S2 and S3). Under treatment of $175 \mathrm{mM} \mathrm{KCl}, 100 \mathrm{mM}$ $\mathrm{Na}_{2} \mathrm{SO}_{4}$, and $300 \mathrm{mM}$ mannitol, the three transgenic lines also exhibited quicker germination than WT (Additional file 1: Figures S4A and S4B; Fig. 3a), and more than $30 \%$ higher cotyledon greening at 14 days for mannitol treatment (Fig. 3b) than WT. However, when treated with $15 \mathrm{mM} \mathrm{LiCl}$, the three transgenic lines exhibited similar seed germination and cotyledon greening to WT (Additional file 1: Figure S4C and S4D). In order to determine whether ectopic expression of GhPLATZ1 alters the expression of the native PLATZ genes in Arabidopsis, the expression levels of 12 members of AtPLATZ genes were determined by qRT-PCR. Eleven 


\section{a}

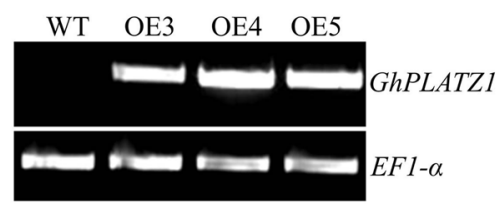

b

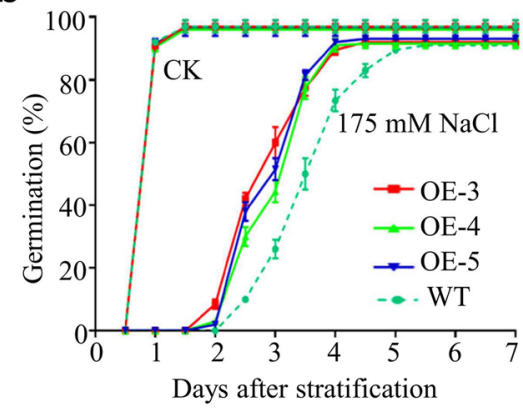

C

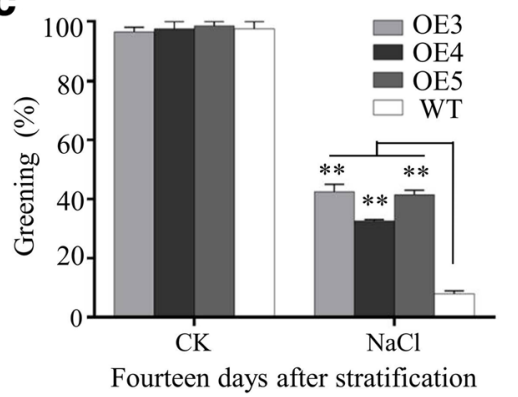

d
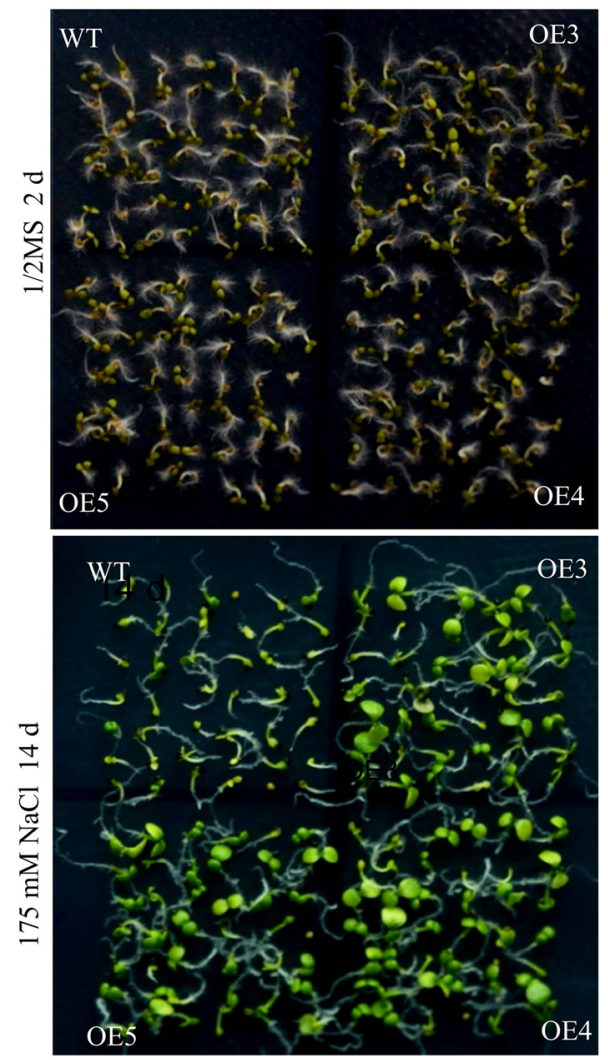

Fig. 2 Phenotypic analysis of GhPLATZ1 transgenic Arabidopsis under salt stress. a Determination of GhPLATZ1 expression in the three independent transgenic $T_{3}$ Arabidopsis homozygous OE3, OE4, and OE5 and WT. b Germination rates of WT, OE3, OE4, and OE5 seeds germinated on 1/2 MS medium with or without $175 \mathrm{mM} \mathrm{NaCl}$ for indicated time points after stratification. c Cotyledon greening of WT, OE3, OE4, and OE5 plants grown on 1/2 MS medium with or without $175 \mathrm{mM} \mathrm{NaCl}$ for 14 days after stratification. d Photographs of 2-day germinated seeds under 1/2 MS medium (upper) and 14-day-old seedlings under 1/2 MS medium containing $175 \mathrm{mM} \mathrm{NaCl}$ (lower). 1/2 MS medium was used as control (CK). Data showed the mean \pm SD $(n=50)$ of three replicates

of the 12 AtPLATZ genes were detected, and their expression levels were not significantly changed in GhPLATZ1 transgenic lines (Additional file 1: Figure S5). However, we did not find a phenotypic difference between GhPLATZ1 transgenic and WT Arabidopsis in vegetative tissues in the presence and absence of salt and mannitol stresses (data not shown). Thus, GhPLATZ1 transgenic lines decreased the sensitivity to osmotic stress during seed germination and postgermination growth.

\section{GhPLATZ1 inhibits ABA biosynthesis}

To investigate whether GhPLATZ1 functions through ABA in transgenic plants, ABA contents were measured. The result showed that dry seeds of GhPLATZ1 transgenic lines contained significantly lower ABA level than WT, whereas germinated seeds of GhPLATZ1 transgenic lines contained similar ABA level to WT at 2.5-day-germination stage with or without $\mathrm{NaCl}$ treatment (Fig. 4a). GhPLATZ1 transgenic lines showed quicker germination than WT only before $2 \mathrm{~d}$ of germination in the presence of exogenous ABA, but similar phenotype after 2 $\mathrm{d}$ of germination (Fig. 4b). ABI4 expression in GhPLATZ1 transgenic lines was down-regulated 2-fold compared with WT at 2.5-day-germination stage with or without $\mathrm{NaCl}$ treatment (Fig. 4c); and $A B I 5$ expression was suppressed by less than two fold (Fig. 4d). These data indicated that GhPLATZ1 inhibited ABA biosynthesis in developing seeds, which might lead to rapid germination in the early stages.

\section{GhPLATZ1 involves GA and ethylene-mediated salt stress responses}

Upon comparing Fig. 5b with Fig. 5a, we found that seed germination was less sensitive to $\mathrm{NaCl}$ after the addition of exogenous GA in both GhPLATZ1 transgenic lines and WT. Under PAC treatment, we observed $40 \%$ lower seed germination at 2-3 days (Fig. 5c), and 30\% lower cotyledon greening at 14 days (Fig. 5d) in WT than in GhPLATZ1 transgenic lines were obtained. Therefore, GhPLATZ1 was involved in the GA-mediated salt stress 


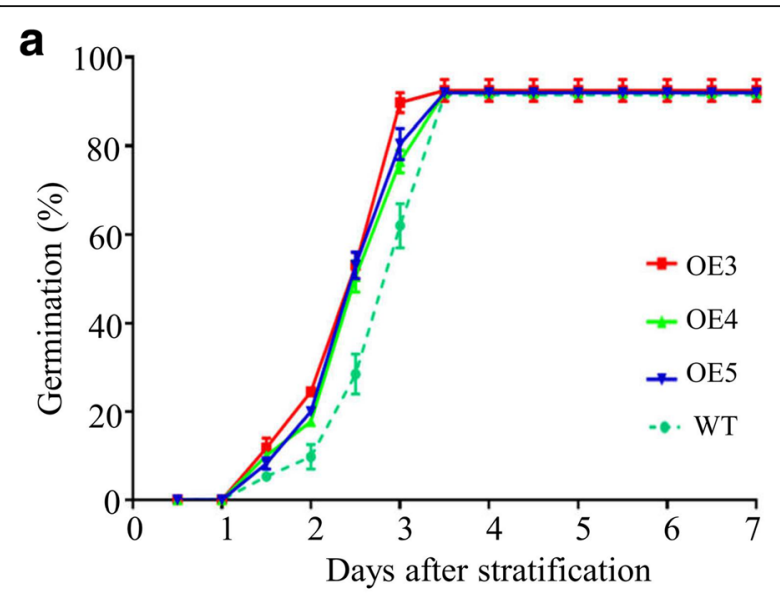

b

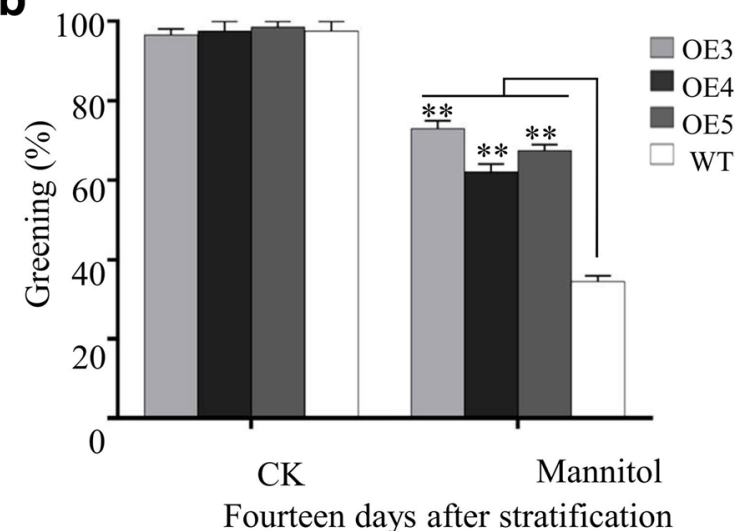

Fig. 3 Phenotypic analysis of GhPLATZ1 in transgenic Arabidopsis under mannitol stress. a Germination rates of WT, OE3, OE4, and OE5 seeds on 1/2 MS medium with $300 \mathrm{mM}$ mannitol for indicated times. b Cotyledon-greening of WT, OE3, OE4, and OE5 plants grown on $1 / 2 \mathrm{MS}$ medium with or without $300 \mathrm{mM}$ mannitol for 14 days. Data show the mean \pm SD $(n=40)$ of three replicates. At least 40 seeds per genotype were measured in each replicate

response of plants during seed germination and postgermination growth.

We also found that seed germination was less sensitive to $\mathrm{NaCl}$ after the addition of exogenous $\mathrm{ACC}$ in both GhPLATZ1 transgenic lines and WT (Fig. 6a). ETO1 (Ethylene-overproduction protein 1) was significantly down-regulated by twofold under salt stress in GhPLATZ1 transgenic lines compared with WT (Fig. 7b). ACS8 was up-regulated by 1.5 times under control and salt stress conditions in GhPLATZ1 transgenic lines compared with WT (Fig. 7c). Thus, GhPLATZ1 was involved in the ethylene-mediated salt stress response in plants during seed germination and postgermination growth.

\section{Discussion}

More than 10 years ago, PLATZ1 was isolated from pea and characterized as a plant-specific zinc-dependent transcription repressor [13]. Recently, only Arabidopsis
PLATZ1 and 2 [19], and maize PLATZ protein FL3 [20] were reported to be involved in abiotic stress and seed development, respectively. Therefore, the roles of PLATZs remain largely unclear.

In the present study, we illustrated that the GhPLATZ1 expression was significantly induced by salt, mannitol, ABA and GA in 20-day-old plants and germinated seeds (Fig. 1a and b), thereby suggesting the involvement of GhPLATZ1 in multiple abiotic stress responses. GhPLATZ1 transgenic lines showed significantly enhanced osmotic, salt, ABA, and PAC insensitivity during seed germination and seedling establishment (Figs. 2, 3, 4 and 5). This function of GhPLATZ1 is similar to that of AtPLATZ1 and AtPLATZ2 whose mutants platz1 and platz2 increased sensitivity to ABA [19]; thus GhPLATZ1 might function similarly to AtPLATZ1 and AtLATZ2 during seed germination. However, GhPLATZ1 transgenic plants showed similar phenotypes to WT in vegetative tissues under normal, salt and mannitol conditions (data not shown), whereas constitutive expression of AtPLATZ1 conferred tolerance to low water availability [19]. Therefore, PLATZs from different species may function similarly in some developing stages but differently in other developing stages in response to abiotic stresses.

Impaired germination and postgermination growth are common among seeds exposed to salt and drought stresses. Plants can regulate and coordinate both growth and/or stress tolerance to promote survival or escape from environmental stress by modifying the production, distribution, or signal transduction of hormones. The hormonal balance between ABA and GA in seed germination in response to environmental stimuli is well documented and discussed [5, 22-24]. ABA plays crucial roles in dormancy induction in developing seeds and in dormancy maintenance in seed imbibition, whereas GAs are involved in germination. Exogenous ABA application inhibits germination in a dose-dependent manner. Applying fluridone, an ABA biosynthesis inhibitor, substantially stimulates seed germination under salinity [1]. The seeds of typical ABA-deficient mutants germinate faster than those of WT [25], and transgenic plants constitutively expressing the $\mathrm{ABA}$ biosynthesis gene maintain considerable seed dormancy $[26,27]$. In contrast, $\mathrm{GA}_{3}$ enhances germination under $\mathrm{NaCl}$ stress [28]. GA-deficient mutants, such as ga1 and ga2, show strong seed dormancy and fail to germinate without exogenous GA treatment [2]. Mutants that are defective in GA 2-oxidases, which deactivate bioactive GA, show decreased seed dormancy [29]. Compared with WT, our data indicated low levels of ABA in dry seeds of GhPLATZ1 transgenic Arabidopsis (Fig. 4a). This result may lead to the insensitivity of GhPLATZ1 transgenic Arabidopsis seeds to osmotic stress and ABA compared with WT. The down-regulation of ABI4 expression (Fig. 4c) confirmed the insensitivity of 


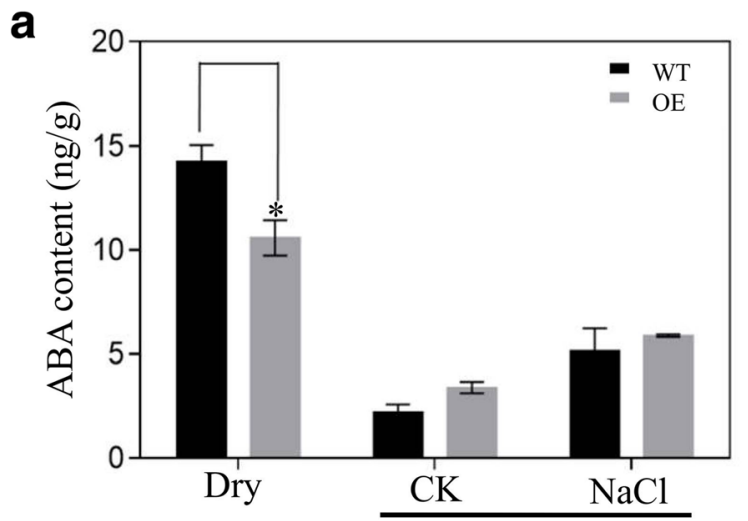

2.5 days after stratification

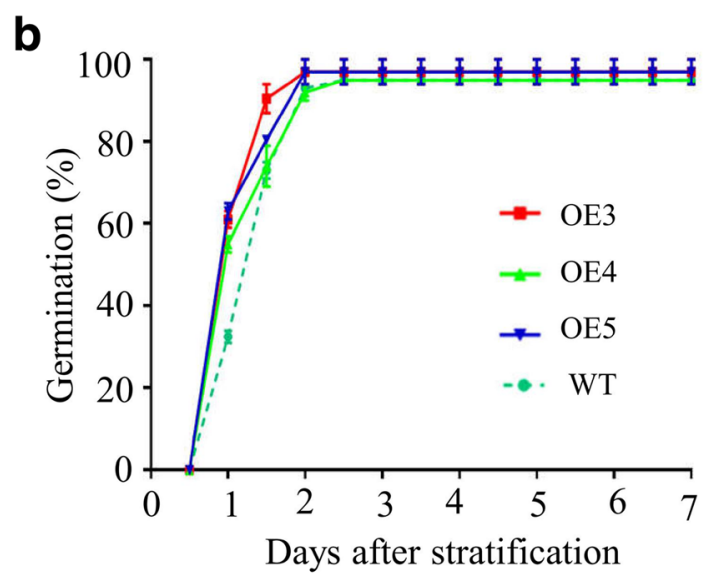

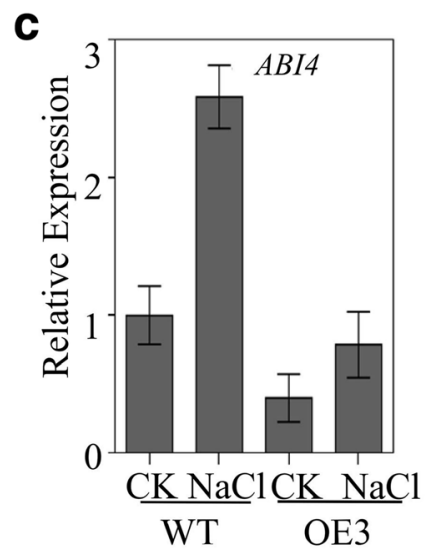

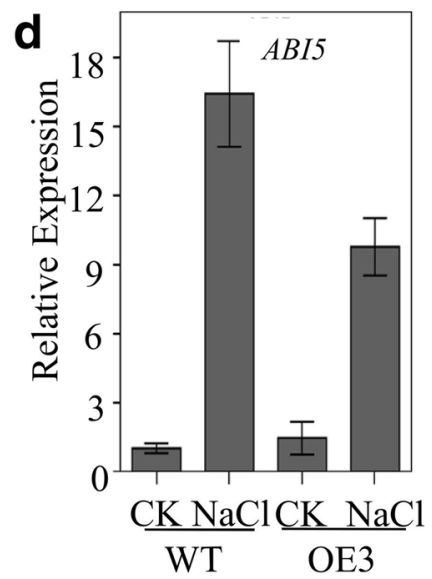

Fig. 4 ABA response of GhPLATZ1 transgenic and WT plants. a Quantification of ABA content in dry seeds and 2.5-day germinated seeds in 1/2 MS medium with or without $175 \mathrm{mM} \mathrm{NaCl}$. b Germination rates of WT, OE3, OE4, and OE5 seeds on 1/2 MS medium containing $5 \mu$ M ABA or indicated times. c The relative expression of $A B / 4$ and $\mathbf{d} A B / 5$ in WT and OE3 seeds germinated for 2 days on $1 / 2$ MS medium with or without $175 \mathrm{mM} \mathrm{NaCl}$. Data show the mean \pm SD $(n=49)$ of three replicates. At least three independent experiments were conducted

GhPLATZ1 transgenic Arabidopsis seeds to osmotic stress and ABA. By contrast, the different responses of GhPLATZ1 transgenic and WT Arabidopsis to PAC indicated the potential high GA in GhPLATZ1 transgenic Arabidopsis in response to salt stress. Therefore, the major active GA forms such as $\mathrm{GA}_{1}$ and $\mathrm{GA}_{4}$ need further investigated. The transcription of a key ABA biosynthetic gene, NCED6, and key GA catabolic gene, $G A 2 o x 7$, is significantly enhanced by $A B I 4$ overexpression by directly binding to their promoters; and ABA induces GA2ox7 transcription whereas GA represses NCED6 expression in an ABI4-dependent manner; and ABA stabilizes the ABI4 protein whereas GA promotes its degradation [30]. Thus, GhPLATZ1 might function in ABA and GA antagonism by suppressing ABI4 transcription and inhibiting the activating expression of NCED6 and GA2ox7, thereby leading to decreased ABA and increased GA (Fig. 7).

In addition, ethylene accelerates seed germination by overcoming the inhibitory action of $\mathrm{ABA}$ or stimulating
GA biosynthesis or its signaling pathway [31]. Two results indicated that GhPLATZ1 increased ethylene production: first, ACS8, a gene that encodes ethylene biosynthesis, showed up-regulated expression in GhPLATZ1 transgenic lines compared with WT (Fig. 6c). $A B I 4$ transcripts were significantly suppressed in GhPLATZ1 transgenic lines (Fig. 4c). Increasing evidence has revealed that ABI4 binds directly to promoters of ACS4, ACS8, and $A C O 2$ to inhibit their transcription causing reduced ethylene production [32]. Therefore, significantly suppressed $A B I 4$ transcripts in GhPLATZ1 transgenic lines suggested the up-regulated expression of ethylene biosynthesis genes, such as ACS4, ACS8, and ACO2. Here, we detected the expression of ACS8, a member of a gene family encoding ethylene biosynthetic enzymes, was up-regulated in GhPLATZ1 in transgenic lines (Fig. 6c). Second, ETO1 showed significantly down-regulated expression in GhPLATZ1 transgenic lines (Fig. 6b). ETO1 is a component of the E3-ligase complex and interacts directly with ACS5 and Le-ACS3 for degradation in a 
a

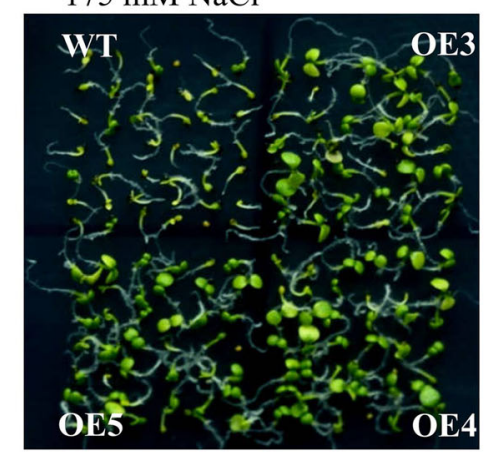

b $175 \mathrm{mM} \mathrm{NaCl}+20 \mu \mathrm{M} \mathrm{GA}$

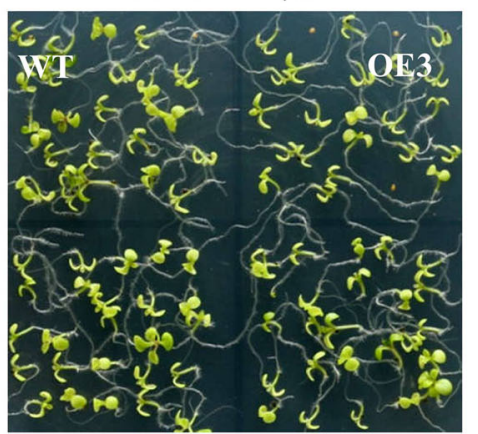

C

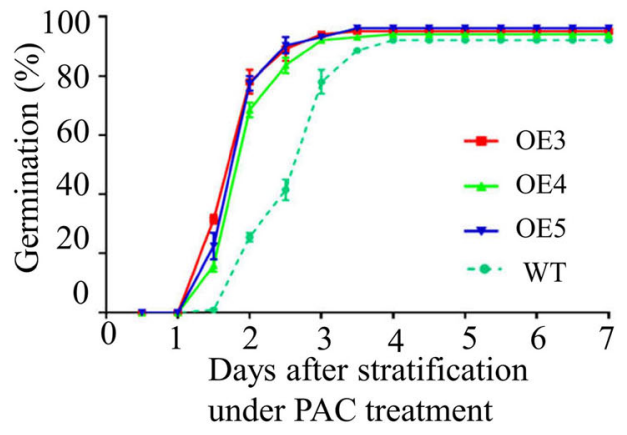

d

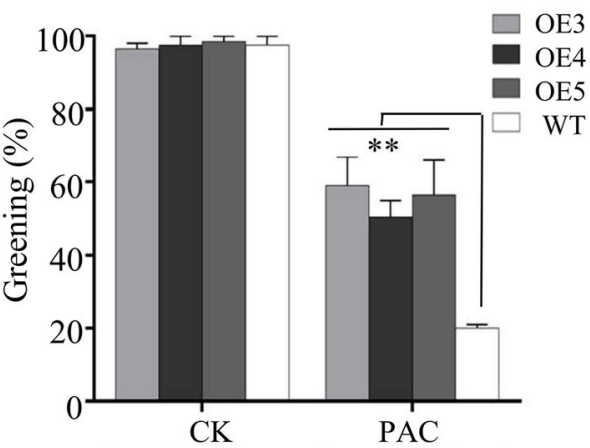

Fourteen days after stratification

Fig. 5 Responses of GhPLATZ1 transgenic and WT plants to GA and PAC. a Photograph of seedlings grown for 14 days on 1/2 MS medium containing $175 \mathrm{mM} \mathrm{NaCl}$ (b) Photograph of seedlings grown for 7 days on 1/2 MS medium containing $175 \mathrm{mM} \mathrm{NaCl}$ and $20 \mu \mathrm{M} \mathrm{GA}$. c Germination rates of WT, OE3, OE4, and OE5 seeds on 1/2 MS medium containing $40 \mu \mathrm{M}$ PAC for indicated times. Data show the mean \pm SD $(n=56)$ of three replicates. $\mathbf{d}$ Cotyledon-greening of WT, OE3, OE4, and OE5 plants grown for 14 days on $1 / 2$ MS medium with or without $40 \mu \mathrm{M}$ PAC. Data show the mean \pm SD $(n=56)$ of three replicates. At least three independent experiments were conducted

proteasome-dependent manner [32-35]. Mutated ETO1 forms result in increased ACS5 protein stability and ethylene overproduction [33]. Therefore, the significantly down-regulation of ETO1 in GhPLATZ1 transgenic lines (Fig. 6b) also suggested increased ethylene levels in GhPLATZ1 transgenic lines. The results of ACC (the direct precursor of ethylene) application (Fig. 7a) further indicated the higher ethylene in GhPLATZ1 transgenic lines than WT. Thus, GhPLATZ1 might increase ethylene production by down-regulating the expression of $A B I 4$ and ETO1 genes (Fig. 7).

\section{Conclusions}

In conclusion, this study discovered a new regulator, GhPLATZ1, which was induced by $\mathrm{NaCl}$, mannitol, ABA and GA treatments. The model in Fig. 7 shows how GhPLATZ1 functions in osmotic stresses during seed germination and seedling establishment. First, GhPLATZ1 increases osmotic insensitivity of transgenic Arabidopsis by inhibiting $A B I 4$ expression, which can induce the expression of NCED6 and GA2ox7. Second, GhPLATZ1 inhibits $A B I 4$ and ETO1 expression, which can suppress $A C S$ gene expression and decrease ACS protein stability, respectively. Therefore, GA and ethylene possibly increased and ABA decreases in GhPLATZ1 transgenic Arabidopsis to promote seed germination and seedling establishment under osmotic stress. However, overexpression of ABI4 and ETO1 in GhPATZ1 transgenic Arabidopsis will confirm this conclusion. However, GhPLATZ1 regulates the expression levels of $A B I 4$ and ETO1, as well as what and how other signaling participate in GhPLATZ1-mediated pathways remains unknown.

\section{Methods}

\section{Plant materials and treatments}

Seeds of upland cotton (Gossypium hirtusium.L) cultivar ZM19 were bought from Chinese Academy of Agricultural Sciences. Seedlings were grown for 20 days in MS liquid medium in a growth chamber with $300 \mu \mathrm{M} \mathrm{m}^{-2} \cdot \mathrm{s}^{-1}$ light intensity and day/night temperatures of $28{ }^{\circ} \mathrm{C} / 20^{\circ} \mathrm{C}$. For each treatment, every 15 uniformly developed seedlings were transferred to MS media containing either $200 \mathrm{mM} \mathrm{NaCl}, 300 \mathrm{mM}$ mannitol, $50 \mu \mathrm{M} \mathrm{ABA}$ or $50 \mu \mathrm{M} \mathrm{GA}_{3}$ for $12 \mathrm{~h}$. In addition, seeds germinated in water for 2.5 days with $0.5 \mathrm{~cm}$ radicles were also treated with MS media containing either $200 \mathrm{mM}$ $\mathrm{NaCl}, 300 \mathrm{mM}$ mannitol, $50 \mu \mathrm{M}$ ABA or $50 \mu \mathrm{M} \mathrm{GA}$ for 


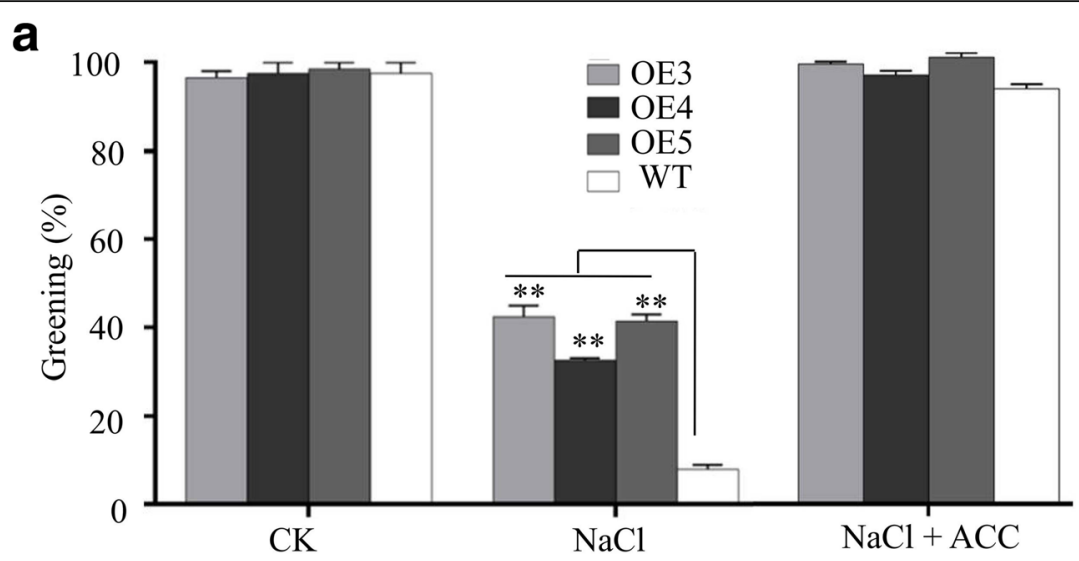

Seven days after stratification

b

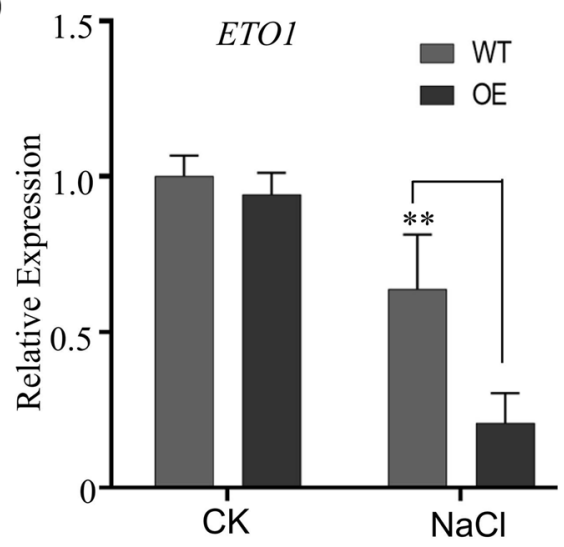

C

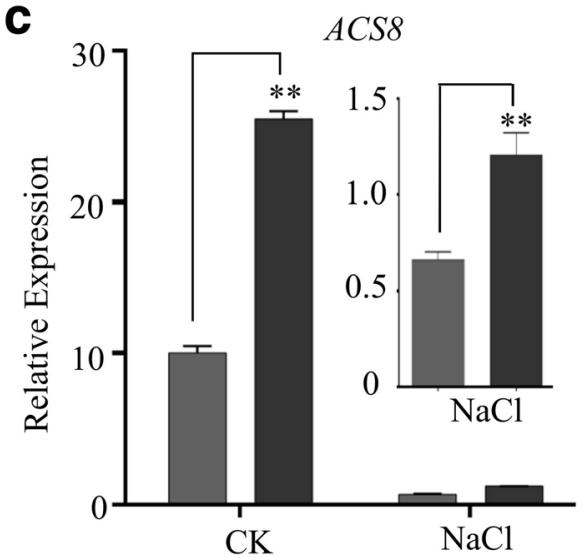

Fig. 6 Responses of GhPLATZ1 transgenic and WT plants to ethylene. a Cotyledon greening of WT, OE3, OE4, and OE5 seedlings grown on 1/2 MS medium with or without $175 \mathrm{mM} \mathrm{NaCl}$, or $175 \mathrm{mM} \mathrm{NaCl}$ and $5 \mu \mathrm{M}$ ACC for 14 days. Data show the mean \pm SD ( $n=49$ ) of three replicates. b The relative expression of ETO1 and c ACS8 in 3.5-d germinated seeds of WT and OE3 on 1/2 MS medium with or without 175 mM NaCl. At least three independent experiments were conducted

6 and $12 \mathrm{~h}$, respectively. Twenty-day seedlings or $2.5-\mathrm{d}$ germinated seeds transferred to MS media were used as control. Samples were harvested at the indicated points, frozen in liquid nitrogen, and stored at $-70{ }^{\circ} \mathrm{C}$ for RNA extraction. Each treatment was repeated three times.

\section{Vector construction and transformation}

To construct 35S::GhPLATZ1, the GhPLATZ1 coding sequence was amplified using cDNA by PCR with gene specific primers (Additional file 2: Table S1). The resulting PCR product was cloned into the SalI and KpnI sites of binary vector pBI121 under the control of a cauliflower mosaic virus $35 \mathrm{~S}$ promoter.

The 35S::GhPLATZ1 was then introduced into Agrobacterium tumefaciens strain GV3101 to be transformed into Arabidopsis thaliana ecotype Columbia-0 by floral dipping [36]. The transgenic plants were screened on $1 / 2 \mathrm{MS}$ medium containing $50 \mathrm{mg} \cdot \mathrm{L}^{-1}$. kanamycin. Two generations of the corresponding $T_{1}$ transgenic seedlings segregated at a ratio of 3:1 (resistant:sensitive) were selected to propagate $\mathrm{T}_{3}$ homozygous. Some of the transgenic Arabidopsis plants were confirmed by detecting the expression of GhPLATZ1 by PCR and used for further experiments. Primers are listed in Additional file 2: Table S1.

\section{RNA extraction}

The total RNAs of different cotton samples used in this report were isolated using RNeasy Plant Mini Kit (QIAGEN). The total RNAs of 20-day-old seedlings and germinated seeds of different Arabidopsis transgenic lines were isolated using a Universal Plant Total RNA Extraction Kit (spin-column)-I (BioTeke Beijing China).

\section{Quantitative RT-PCR}

qRT-PCR was performed as previously described [37]. Cotton GhUBI (EU604080) or Arabidopsis actin2 genes were used as the standard control. The relative GhPLATZ1 and AtPLATZs expression level was analyzed using the comparative CT method, and three replicates of each sample were analyzed. All of the primers used are listed 


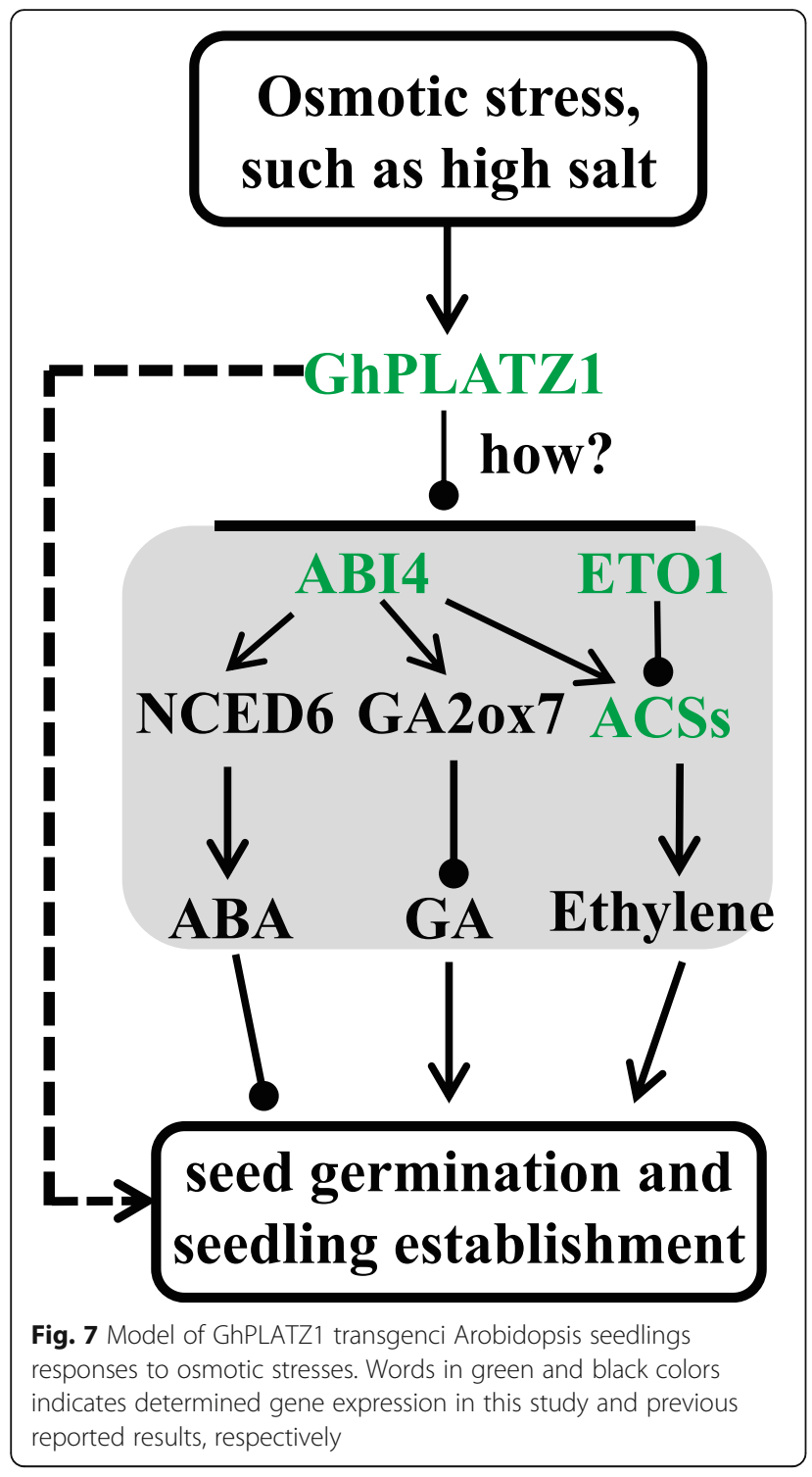

in Additional file 2: Table S1. At least three independent experiments were carried out.

\section{Fluorescence microscopy}

Roots from seven-day-old transgenic Arabidopsis containing 35S::GhPL-GFP were used and imaged by LSCM51 (Zeiss) at $488 \mathrm{~nm}$ for GFP.

\section{Seed germination and cotyledon greening analysis}

Seeds of each genotype were harvested from plants of the same age and stored for 4 weeks in the dark at $4{ }^{\circ} \mathrm{C}$. For each comparison, seeds were surface-sterilized with $70 \%$ ethanol for $5 \mathrm{~min}$, subsequently incubated in $2.6 \%$ sodium hypochlorite for $10 \mathrm{~min}$, and washed five times with sterile water. At least 30 sterile seeds were plated on $1 / 2$ MS medium plus $1 \%(w / v)$ sucrose with or without different concentrations of $\mathrm{NaCl}, \mathrm{KCl}, \mathrm{Na}_{2} \mathrm{SO}_{4}, \mathrm{LiCl}$, mannitol, ABA, PAC, or $\mathrm{NaCl}$ combined with GA or ACC as indicated. Plates were chilled at $4{ }^{\circ} \mathrm{C}$ in the dark for 3 days (stratification) and moved to $22{ }^{\circ} \mathrm{C}$ with a 16-h-light/8-h-dark cycle. The percentage of seed germination was determined at indicated time points. Germination was defined as an obvious emergence of the radicle through the seed coat. The percentage of cotyledon greening was recorded at 14 days after the end of stratification. Cotyledon greening was defined as obvious cotyledon expansion and turning of cotyledon color into green. Three biological replications were performed.

\section{ABA content measurements}

To quantify ABA content, dry seeds and two-day germinated seeds of GhPLATZ1 overexpressing Arabidopsis and WT treated with or without $175 \mathrm{mM} \mathrm{NaCl}$ were ground in liquid nitrogen. Furthermore, $150 \mathrm{mg}$ of seed powder was homogenized and extracted for $24 \mathrm{~h}$ in methanol containing D6-ABA (OIChemIm Co., Ltd.) as an internal standard. The mixture was purified with an Oasis Max solid-phase extract cartridge (150 mg/6 cc; waters) and eluted with $5 \%$ formic acid in methanol. The elution was dried and reconstituted. ABA content was measured by HPLC-MS-MS (Agilent 1290 Rapid-resolution Liquid chromatography, Agilent Technologies, Waldbronn, Germany; Sciex 6500 Q-Trap AB Technologies, USA). The mobile phase consisted of A:B (methyl alcohol/0.1\% methanoic acid: $\mathrm{H}_{2} \mathrm{O} /$ $0.1 \%$ methanoic acid). The elution gradient was $0-2 \mathrm{~min}$, $A=20 \%$; 2-14 $\mathrm{min}, A=80 \%$; and $15.1-20 \mathrm{~min}, A=20 \%$. The injection volume was $2 \mu \mathrm{L}$. The mass spectrometer conditions were as follows: spray voltage, $4500 \mathrm{~V}$; atomizer pressure, $65 \mathrm{psi}$; assist device pressure, $70 \mathrm{psi}$; atomization temperature, $400{ }^{\circ} \mathrm{C}$.

\section{Statistical analysis}

Data were subjected to data processing system and significant differences were established through one-way ANOVA. Differences at $5 \%$ and $1 \%$ levels were considered significant and denoted by single and double stars $\left({ }^{*} P<0.05 ;{ }^{* *} P<0.01\right)$.

\section{Additional files}

Additional file 1: Figure S1. Sequence analysis of GhPLATZ1 (GenBank accession no. AFH57272) with its homologs. (A) Multiple alignments of GhPLATZs. Identical amino acids are shaded in black. The conserved zinc-fingers are indicated by $\mathrm{C}$ and $\mathrm{H}$ at the bottom of the sequences. (B) Phylogenetic analysis of GhPLATZs and AtPLATZs. The GenBank accession numbers are as follows: GhPLATZ1 (XP_016742770.1), GhPLATZ2 (XP_016736692.1), GhPLATZ3 (XP_016705363.1), GhPLATZ4 (XP_01672128.1), GhPLATZ5 (XP_012488860.1), GhPLATZ6 (XP_016670611.1), GhPLATZ7 (XP_016723269.1), GhPLATZ8 (XP_016728634.1), GhPLATZ9 (XP_016742383.1), AtPLATZ1.1 (AT1G21000.1), AtPLATZ1.2 (AT1G21000.2), AtPLATZ2 (AT1G76590.1), AtPLATZ3.1 (AT1G32700.1), AtPLATZ3.2 (AT1G32700.2), AtPLATZ4 (AT1G43000.1), AtPLATZ5 (AT1G31040.1), AtPLATZ6 (AT2G01818.1), 
AtPLATZ7 (AT2G12646.1), AtPLATZ8 (AT2G27930.1), AtPLATZ9 (AT3G50808.1), AtPLATZ10 (AT3G60670.1), AtPLATZ11.1 (AT4G17900.1), AtPLATZ11.2 (T4G17900.2), AtPLATZ12 (AT5G46710.1). Figure S2.

Phenotypes of GhPLATZ1 transgenic Arabidopsis under salt conditions. (A) Photographs taken at 3,5, and $7 \mathrm{~d}$ on 1/2 MS medium containing different concentrations of $\mathrm{NaCl}$. (B) Germination rates of WT and OE-3 seeds on $1 / 2$ MS medium with or without 175 or $200 \mathrm{mM} \mathrm{NaCl}$ in (A). (C) Cotyledon greening of WT and OE-3 seedlings grown on 1/2 MS medium with or without 150, 175 or $200 \mathrm{mM} \mathrm{NaCl}$ in (A). Figure S3. Phenotypic analysis of WT and GhPLATZ1 transgenic Arabidopsis seeds on 1/2 MS medium for $3.5 \mathrm{~d}(\mathrm{~A})$ and $1 / 2 \mathrm{MS}$ medium with or without $200 \mathrm{mM} \mathrm{NaCl}$ for14 d (B). Figure S4. Phenotypes of GhPLATZ1 transgenic Arabidopsis under potassium, sodium and lithium stresses. Germination rates of WT, $\mathrm{OE}-3, \mathrm{OE}-4$, and $\mathrm{OE}-5$ seeds on 1/2 MS medium with or without $175 \mathrm{mM}$ $\mathrm{KCl}$ (A), $100 \mathrm{mM} \mathrm{Na}_{2} \mathrm{SO}_{4}$ (B), and $15 \mathrm{mM} \mathrm{LiCl}$ (C) for indicated times. (D) Cotyledon-greening of WT, OE-3, OE-4, and OE-5 seedlings grown on 1/2 MS medium with or without $15 \mathrm{mM} \mathrm{LiCl}$ for 14 days. Figure S5. Expression of AtPLATZ genes in GhPLATZ1 transgenic and WT plants. AtPLATZ1 (AT1G210001), AtPLATZ2 (AT1G76590.1), AtPLATZ3.1 (AT1G32700.1), AtPLATZ4 (AT1G43000.1), AtPLATZ5 (AT1G31040.1), AtPLATZ6 (AT2G01818.1), AtPLATZ7 (AT2G12646.1), AtPLATZ8 (AT2G27930.1), AtPLATZ9 (AT3G50808.1), AtPLATZ10 (AT3G60670.1), AtPLATZ11.1 (AT4G17900.1), AtPLATZ12 (AT5G46710.1). Primers used here are listed in Additional file 2: Table S1. (DOCX $21 \mathrm{~kb}$ )

Additional file 2: Table S1. List of primers used in this study. (DOCX 21 kb)

\section{Abbreviations}

ABA: Abscisic acid; ABI: ABA insensitive; ACC: 1-aminocyclopropane-1carboxylic acid; ACS: ACC synthase; CK: Control; ETO1: Ethyleneoverproduction protein 1; GA: Gibberellin; MS: Murashige and Skoog; OE: Transgenic arabidopsis with high GhPLATZ1 overexpression; PAC: Paclobutrazol; WT: Wild type

\section{Acknowledgments}

None.

\section{Funding}

This work was supported by the National Natural Science Foundation of China (Grant number 31471425), the Natural Science Foundation of Shandong Province (Grant number ZR2015CM002).

\section{Availability of data and materials}

The data sets supporting the results of this article are included within the article and its additional files. Sequence data used in this manuscript can be found in the Arabidopsis Information Resource (TAIR, https://www.arabidopsis.org/servlets/ Search?type=general\&search_action $=$ detail\&method $=1$ \&show_obsolete $=$ F\&name=PLATZ\&sub_type=gene\&SEARCH_EXACT=4\&SEARCH_CONTAINS=1) and The National Center for Biotechnology Information (https://blast.ncbi.nIm. nih.gov/Blast.cgi), under the following accession numbers: GhPLATZ1 (XP_016742770.1), GhPLATZ2 (XP_016736692.1), GhPLATZ3 (XP_016705363.1), GhPLATZ4 (XP_01672128.1), GhPLATZ5 (XP_012488860.1), GhPLATZ6 (XP_016670611.1), GhPLATZ7 (XP_016723269.1), GhPLATZ8 (XP_016728634.1), GhPLATZ9 (XP_016742383.1), AtPLATZ1.1 (AT1G21000.1), AtPLATZ1.2 (AT1G21000.2), AtPLATZ2 (AT1G76590.1), AtPLATZ3.1 (AT1G32700.1), AtPLATZ3.2 (AT1G32700.2), AtPLATZ4 (AT1G43000.1), AtPLATZ5 (AT1G31040.1), AtPLATZ6 (AT2G01818.1), AtPLATZ7 (AT2G12646.1), AtPLATZ8 (AT2G27930.1), AtPLATZ9 (AT3G50808.1), AtPLATZ10 (AT3G60670.1), AtPLATZ11.1 (AT4G17900.1), AtPLATZ11.2 (T4G17900.2), AtPLATZ12 (AT5G46710.1).

\section{Authors' contributions}

SCZ identified voucher specimen and carried out Arabidopsis transformation, majority of molecular studies and data analysis. SCZ also drafted the manuscript. RY, YQH, and SSL participated in phenotypic analysis, qRT-PCR and references editing. GDY, JGH and CCZ give some advices for experimental design. CAW conceived the study, participated in the design and coordination of this study and drafted the manuscript. All of the authors read and approved the final manuscript.

\section{Ethics approval and consent to participate}

Seeds of upland cotton (Gossypium hirtusium.L) cultivar ZM19 were bought from Chinese Academy of Agricultural Sciences.

\section{Consent for publication}

Not applicable.

\section{Competing interests}

The authors declare that they have no competing interests.

\section{Publisher's Note}

Springer Nature remains neutral with regard to jurisdictional claims in published maps and institutional affiliations.

\section{Received: 31 October 2017 Accepted: 5 September 2018}

Published online: 04 October 2018

\section{References}

1. Finkelstein RR, Gampala SS, Rock CD. Abscisic acid signaling in seeds and seedlings. Plant Cell. 2002;14(Suppl):S15-45.

2. Shu K, Zhang H, Wang S, Chen M, Wu Y, Tang S, Liu C, Feng Y, Cao X, Xie $Q$. ABI4 regulates primary seed dormancy by regulating the biogenesis of abscisic acid and gibberellins in arabidopsis. PLoS Genet. 2013;9:e1003577.

3. Finkelstein RR, Lynch TJ. The Arabidopsis abscisic acid response gene ABI5 encodes a basic leucine zipper transcription factor. Plant Cell. 2000;12:599-609.

4. Lopez-Molina L, Mongrand S, Chua NH. A postgermination developmental arrest checkpoint is mediated by abscisic acid and requires the ABI5 transcription factor in Arabidopsis. Proc Natl Acad Sci U S A. 2001;98:4782-7

5. Shu K, Liu XD, Xie Q, He ZH. Two faces of one seed: hormonal regulation of dormancy and germination. Mol Plant. 2016;9:34-45.

6. J.M. Daviere, P. Achard, Gibberellin signaling in plants, development (Cambridge, England), 140 (2013) 1147-1151.

7. Sun TP, Gubler F. Molecular mechanism of gibberellin signaling in plants. Annu Rev Plant Biol. 2004;55:197-223.

8. Dill A, Thomas SG, Hu J, Steber CM, Sun TP. The Arabidopsis F-box protein SLEEPY1 targets gibberellin signaling repressors for gibberellin-induced degradation. Plant Cell. 2004;16:1392-405.

9. Wang Y, Liu C, Li K, Sun F, Hu H, Li X, Zhao Y, Han C, Zhang W, Duan Y, Liu M, Li X. Arabidopsis EIN2 modulates stress response through abscisic acid response pathway. Plant Mol Biol. 2007:64:633-44.

10. Pirrello J, Jaimes-Miranda F, Sanchez-Ballesta MT, Tournier B, Khalil-Ahmad Q, Regad F, Latche A, Pech JC, Bouzayen M. SI-ERF2, a tomato ethylene response factor involved in ethylene response and seed germination. Plant \& cell physiology. 2006:47:1195-205.

11. Kielbowicz-Matuk A. Involvement of plant $\mathrm{C}(2) \mathrm{H}(2)$-type zinc finger transcription factors in stress responses. Plant science : an international journal of experimental plant biology. 2012;185-186:78-85.

12. Riechmann JL, Heard J, Martin G, Reuber L, Jiang C, Keddie J, Adam L, Pineda O, Ratcliffe OJ, Samaha RR, Creelman R, Pilgrim M, Broun P, Zhang JZ, Ghandehari D, Sherman BK, Yu G. Arabidopsis transcription factors: genome-wide comparative analysis among eukaryotes, science (New York). NY. 2000;290:2105-10.

13. Nagano Y, Furuhashi H, Inaba T, Sasaki Y. A novel class of plant-specific zincdependent DNA-binding protein that binds to a/T-rich DNA sequences. Nucleic Acids Res. 2001:29:4097-105.

14. Schwabe JW, Klug A. Zinc mining for protein domains. Nat Struct Biol. 1994; $1: 345-9$.

15. Teakle GR, Manfield IW, Graham JF, Gilmartin PM. Arabidopsis thaliana GATA factors: organisation, expression and DNA-binding characteristics. Plant Mol Biol. 2002;50:43-57.

16. Manfield IW, Devlin PF, Jen CH, Westhead DR, Gilmartin PM. Conservation, convergence, and divergence of light-responsive, circadian-regulated, and tissue-specific expression patterns during evolution of the Arabidopsis GATA gene family. Plant Physiol. 2007;143:941-58.

17. Griffiths S, Dunford RP, Coupland G, Laurie DA. The evolution of CONSTANS like gene families in barley, rice, and Arabidopsis. Plant Physiol. 2003;131: 1855-67. 
18. Noguero M, Atif RM, Ochatt S, Thompson RD. The role of the DNA-binding one zinc finger (DOF) transcription factor family in plants. Plant science : an international journal of experimental plant biology. 2013;209:32-45.

19. Gonzalez-Morales SI, Chavez-Montes RA, Hayano-Kanashiro C, Alejo-Jacuinde G, Rico-Cambron TY, de Folter S, Herrera-Estrella L. Regulatory network analysis reveals novel regulators of seed desiccation tolerance in Arabidopsis thaliana. Proc Natl Acad Sci U S A. 2016;113:E5232-41.

20. Li Q, Wang J, Ye J, Zheng X, Xiang X, Li C, Fu M, Wang Q, Zhang Z, Wu Y. The maize imprinted gene floury 3 encodes a PLATZ protein required for tRNA and $5 \mathrm{~S}$ rRNA transcription through interaction with RNA polymerase III. Plant Cell. 2017; https://doi.org/10.1105/tpc.17.00576.

21. Wang G, Zhu Q, Meng Q, Wu C. Transcript profiling during salt stress of young cotton (Gossypium hirsutum) seedlings via Solexa sequencing. Acta Physiol Plant. 2012;34:107-15.

22. Finkelstein $R$, Reeves $W$, Ariizumi T, Steber $C$. Molecular aspects of seed dormancy. Annu Rev Plant Biol. 2008:59:387-415.

23. Rajjou L, Duval M, Gallardo K, Catusse J, Bally J, Job C, Job D. Seed germination and vigor. Annu Rev Plant Biol. 2012;63:507-33.

24. Colebrook EH, Thomas SG, Phillips AL, Hedden P. The role of gibberellin signalling in plant responses to abiotic stress. J Exp Biol. 2014;217:67-75.

25. Frey A, Effroy D, Lefebvre V, Seo M, Perreau F, Berger A, Sechet J, A. To, North HM, Marion-Poll A. Epoxycarotenoid cleavage by NCED5 fine-tunes ABA accumulation and affects seed dormancy and drought tolerance with other NCED family members. The Plant journal : for cell and molecular biology. 2012;70:501-12.

26. Martinez-Andujar C, Ordiz Ml, Huang Z, Nonogaki M, Beachy RN, Nonogaki H. Induction of 9-cis-epoxycarotenoid dioxygenase in Arabidopsis thaliana seeds enhances seed dormancy. Proc Natl Acad Sci U S A. 2011;108:17225-9.

27. Nonogaki M, Sall K, Nambara E, Nonogaki H. Amplification of ABA biosynthesis and signaling through a positive feedback mechanism in seeds. The Plant journal : for cell and molecular biology. 2014;78:527-39.

28. Atia A, Debez A, Barhoumi Z, Smaoui A, Abdelly C. ABA, GA(3), and nitrate may control seed germination of Crithmum maritimum (Apiaceae) under saline conditions. Comptes rendus biologies. 2009;332:704-10.

29. Yamauchi Y, Takeda-Kamiya N, Hanada A, Ogawa M, Kuwahara A, Seo M, Kamiya $Y$, Yamaguchi S. Contribution of gibberellin deactivation by AtGA20x2 to the suppression of germination of dark-imbibed Arabidopsis thaliana seeds. Plant \& cell physiology. 2007;48:555-61.

30. Shu K, Chen Q, Wu Y, Liu R, Zhang H, Wang P, Li Y, Wang S, Tang S, Liu C, Yang W, Cao X, Serino G, Xie Q. ABI4 mediates antagonistic effects of abscisic acid and gibberellins at transcr ipt and protein levels. Plant J. 2016; 85:348-61.

31. Dong Z, Yu Y, Li S, Wang J, Tang S, Huang R. Abscisic acid antagonizes ethylene production through the ABI4-mediated transcriptional repression of ACS4 and ACS8 in Arabidopsis. Mol Plant. 2016;9:126-35.

32. Corbineau F, Xia Q, Bailly C, El-Maarouf-Bouteau H. Ethylene, a key factor in the regulation of seed dormancy. Front Plant Sci. 2014;5:539.

33. Wang KL, Yoshida H, Lurin C, Ecker JR. Regulation of ethylene gas biosynthesis by the Arabidopsis ETO1 protein. Nature. 2004;428:945-50.

34. M.J. Christians, D.J. Gingerich, M. Hansen, B.M. Binder, J.J. Kieber, R.D. Vierstra, The BTB ubiquitin ligases ETO1, EOL1 and EOL2 act collectively to regulate ethylene biosynthesis in Arabidopsis by controlling type-2 ACC synthase levels, Plant J : for cell and molecular biology, 57 (2009) 332-345.

35. Yoshida H, Nagata M, Saito K, Wang KL, Ecker JR. Arabidopsis ETO1 specifically interacts with and negatively regulates type 21 aminocyclopropane-1-carboxylate synthases. BMC Plant Biol. 2005;5:14

36. Clough SJ, Bent AF. Floral dip: a simplified method for agrobacteriummediated transformation of Arabidopsis thaliana. The Plant journal : for cell and molecular biology. 1998;16:735-43.

37. Chen C, Wu C, Miao J, Lei Y, Zhao D, Sun D, Yang G, Huang J, Zheng C. Arabidopsis SAG protein containing the MDN1 domain participates in seed germination and seedling development by negatively regulating $A B \mid 3$ and ABI5. J Exp Bot. 2014;65:35-45.

Ready to submit your research? Choose BMC and benefit from:

- fast, convenient online submission

- thorough peer review by experienced researchers in your field

- rapid publication on acceptance

- support for research data, including large and complex data types

- gold Open Access which fosters wider collaboration and increased citations

- maximum visibility for your research: over $100 \mathrm{M}$ website views per year

At $\mathrm{BMC}$, research is always in progress.

Learn more biomedcentral.com/submissions 